Old Dominion University

ODU Digital Commons

Physics Faculty Publications

Physics

2015

\title{
Measurements of Population Densities of Metastable and Resonant Levels of Argon Using Laser Induced Fluorescence
}

\author{
M. Nikolic \\ Old Dominion University \\ J. Newton \\ Old Dominion University \\ C. I. Sukenik \\ Old Dominion University, csukenik@odu.edu \\ L. Vuskovic \\ Old Dominion University, lvuskovi@odu.edu \\ S. Popovic \\ Old Dominion University
}

Follow this and additional works at: https://digitalcommons.odu.edu/physics_fac_pubs

Part of the Atomic, Molecular and Optical Physics Commons

\section{Repository Citation}

Nikolic, M.; Newton, J.; Sukenik, C. I.; Vuskovic, L.; and Popovic, S., "Measurements of Population Densities of Metastable and Resonant Levels of Argon Using Laser Induced Fluorescence" (2015). Physics Faculty Publications. 2.

https://digitalcommons.odu.edu/physics_fac_pubs/2

\section{Original Publication Citation}

Nikolic, M., Newton, J., Sukenik, C.I., Vuskovic, L., \& Popovic, S. (2015). Measurements of population densities of metastable and resonant levels of argon using laser induced fluorescence. Journal of Applied Physics, 117(2), 1-8. doi: 10.1063/1.4905611

This Article is brought to you for free and open access by the Physics at ODU Digital Commons. It has been accepted for inclusion in Physics Faculty Publications by an authorized administrator of ODU Digital Commons. For more information, please contact digitalcommons@odu.edu. 


\title{
Measurements of population densities of metastable and resonant levels of argon using laser induced fluorescence
}

\author{
M. Nikolić, J. Newton, C. I. Sukenik, L. Vušković, and S. Popović \\ Center for Accelerator Science, Department of Physics, Old Dominion University, Norfolk, Virginia 23529 , \\ USA
}

(Received 12 May 2014; accepted 26 December 2014; published online 13 January 2015)

\begin{abstract}
We present a new approach to measure population densities of Ar I metastable and resonant excited states in low temperature Ar plasmas at pressures higher than 1 Torr. This approach combines the time resolved laser induced fluorescence technique with the kinetic model of Ar. The kinetic model of Ar is based on calculating the population rates of metastable and resonant levels by including contributions from the processes that affect population densities of Ar I excited states. In particular, we included collisional quenching processes between atoms in the ground state and excited states, since we are investigating plasma at higher pressures. We also determined time resolved population densities of Ar I $2 p$ excited states by employing optical emission spectroscopy technique. Time resolved Ar I excited state populations are presented for the case of the postdischarge of the supersonic flowing microwave discharge at pressures of 1.7 and 2.3 Torr. The experimental set-up consists of a pulsed tunable dye laser operating in the near infrared region and a cylindrical resonance cavity operating in $\mathrm{TE}_{111}$ mode at $2.45 \mathrm{GHz}$. Results show that time resolved population densities of Ar I metastable and resonant states oscillate with twice the frequency of the discharge. (C) 2015 AIP Publishing LLC. [http://dx.doi.org/10.1063/1.4905611]
\end{abstract}

\section{INTRODUCTION}

Low temperature, non-equilibrium gas discharge plasmas used for the processing of solid surfaces have been attracting ongoing attention. Indeed, since the development of the semiconductor industry, non-equilibrium plasma processing utilizing various types of gas discharges has become an essential part of modern technology. There are many important applications of non-equilibrium gas discharges in the processing of the semiconductors such as sputtering, plasma enhanced chemical vapor deposition, and plasma etching and cleaning processes. ${ }^{1-6}$ Even though these discharges are widely used, knowledge of the fundamental physical and chemical phenomena which determine the outcome of the plasma surface interactions is mostly empirical. Therefore, developing models and experiments to improve understanding, performance, and control of these processing plasmas is greatly needed.

One of the key characteristics in low-temperature gas discharges is the kinetics of excited particles since the study of the kinetics of excited particles can lead to better understanding of radiative and chemical properties of discharges and improvement of their application. ${ }^{7,8}$ Especially important are atoms in metastable states, which play an important role in characterization of gas discharges. Due to their high energy and long life time, they are an important energy reservoir, which can be transmitted to the rest of plasma particles by collisional processes.

For all these reasons, characterization of atoms in metastable states using plasma diagnostic techniques is of fundamental importance. Commonly used diagnostic methods, such as the Langmuir probe method, may not be applicable for microwave (MW) plasmas diagnostics because the metal object in the MW cavity affects the field distribution and shifts resonant frequency. An alternative choice is Optical Emission Spectroscopy (OES) since it is in situ, nonintrusive, easy to use, and an inexpensive technique. However, OES is useful only when detecting species that are excited to energy levels higher than ground and metastable states. Radiative decay of metastable levels is electric dipole forbidden and they decay back to the ground state mostly through non-radiative collisional quenching processes. Laser based plasma diagnostics, such as Laser Induced Fluorescence (LIF), represents a complementary technique that allows in situ measurements of population densities of ground and metastable atoms. Moreover, applying LIF increases the overall precision of data collection. A variety of indirect and direct methods have been developed to obtain these population densities. Direct measurements of metastable population densities include laser induced fluorescence measurements ${ }^{9,10}$ or absorption measurements. ${ }^{11}$ Indirect measurements include a detailed modelling of production and loss mechanisms of a specific Ar level. ${ }^{12-15}$

Here, we present a new approach to study the time resolved population densities of Ar metastable and resonant levels by employing the LIF technique in combination with the kinetic model of argon. This approach relies on an assumption that the difference in spectral line intensities when the system is pumped by a laser and without a laser is directly proportional to the population densities of the $s$-states. In particular, we focused on metastable Ar I $1 s_{5}\left(4 s[3 / 2]_{2}{ }^{3} P_{2} J=2\right)$ state obtained from the spectral line intensity at $706.72 \mathrm{~nm}$ and resonant $1 s_{4}\left(4 s[3 / 2]_{1}{ }^{3} P_{1} J=1\right)$ state obtained from the spectral line intensity at $727.29 \mathrm{~nm}$. In order to prove this assumption, we have developed a full kinetic model of Ar based on calculating the population rates of metastable and 
resonant levels by including contributions from all the processes that affect the populations of the desired states. We also compared the results with population densities obtained using plasma tomography methods. ${ }^{16}$

In Secs. II-VI, we briefly describe the experimental setup, a cavity MW discharge in the supersonic flow, and the pulsed dye laser system. Then we provide the detailed description of the kinetic model and developed technique for evaluation of time resolved population densities of $\mathrm{Ar}$ I excited states. Finally, we present and discuss the experimental results.

\section{EXPERIMENTAL SET-UP}

The experiment was conducted in a supersonic flowing tube, combined with a microwave cavity discharge, shown in Fig. 1. A commercial microwave generator, operating in the S-band at $2.45 \mathrm{GHz}$, was used to sustain a cylindrical cavity discharge at power density of up to $4 \mathrm{~W} / \mathrm{cm}^{3}$. The discharge is generated inside a cylindrical quartz tube with the inner diameter of $32 \mathrm{~mm}$. The working pressure in the evacuated quartz tube was kept between 1 and 3 Torr. A Mach 2, cylindrical convergent-divergent De Laval nozzle was used to sustain the supersonic flowing discharge downstream of the microwave cavity, which operated in the $\mathrm{TE}_{111}$ mode. The measurements were performed in pure argon that was fed into the stagnation chamber through a gas manifold. Gas flow was established by a roots blower (Pfeiffer Okta $500 \mathrm{~A}$ ), which was backed by two roughing pumps (Varian SD-700). The capacity of the pumping system allowed for generation of supersonic flow at static pressures of 1-20 Torr. A detailed explanation of the experimental set-up is given in Ref. 17.

In the post discharge region of the pure Ar discharge, a plasmoid-like formation was observed as a secondary downstream phenomenon coupled to the microwave cavity. The plasmoid appears to be sustained by a low power surface wave, which propagates along its surface and the surface of the quartz tube. Our initial analysis led to the conclusion that plasmoid formation may be caused by aerodynamic effects in the supersonic flow. The full interpretation of the origins of the plasmoid object requires knowledge of all effects causing its formation.

In order to clarify the heavy-particle temperature evolution in the flow, we note that the experiment is divided into three heating sections, which occupy about $40 \mathrm{~cm}$ of the

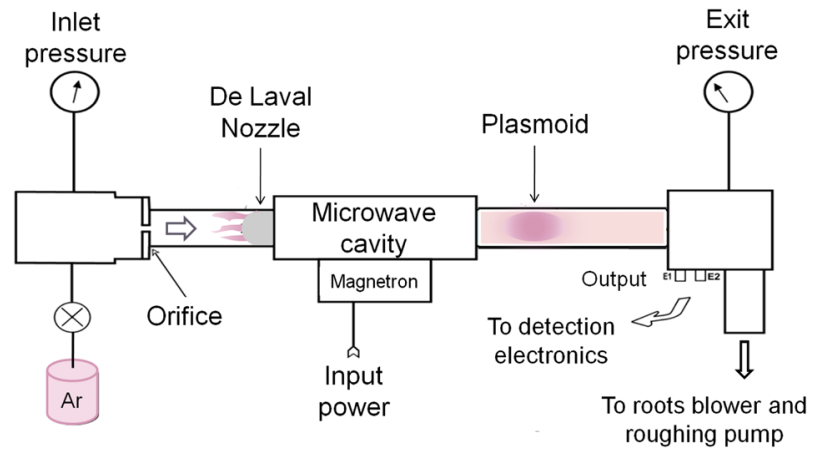

FIG. 1. Scheme of the supersonic flowing microwave discharge. tube. They all contributed to the heating of heavy particles and are arranged along the flow tube as follows. First section is about $2 \mathrm{~cm}$ long converging part of the nozzle, where the ionization effects were protruding upstream into highpressure gas, which was moving slowly toward the throat. In this section, the discharge had the filamentary pattern, due to the high-pressure thermal ionization instabilities. This section was followed by the second section consisting of the primary microwave discharge, which was generated inside the microwave cavity, as indicated in Figure 2. It was about $25 \mathrm{~cm}$ long and included the diverging part of the nozzle, which was made of high temperature ceramics. By far the most power was dissipated in this second section. The center of the third section, where the LIF measurement took place, was located about $12 \mathrm{~cm}$ downstream. Gas temperature measurements were performed earlier at the end of the second, microwave cavity section and referred in Ref. 18. All heating sections were powered from the same alternating power source, which produced the train of microwave pulses with duration of about $8 \mathrm{~ms}$. Flight history of an individual heavy particle, from the converging through the diverging parts of the nozzle and the microwave cavity discharge to the point of measurement referred in Ref. 18 was about $10 \mathrm{~ms}$ long. Time of flight of the particle from the exit of the cavity to the measurement point was about $200 \mu$ s, and its variation was not more than $50 \mathrm{~K}$. Therefore, the gas particles entering the measurement point were already hot and were not additionally heated by the plasmoid to the error that we estimate at $10 \%$, which is more than in Ref. 18 . We overestimate the temperature error, since we did not perform simultaneous measurement of temperature to the LIF. Therefore, the temperature fluctuations and their consequences in the third section were small, especially due to the smaller power dissipation in comparison to the microwave cavity discharge.

We employed optical emission spectroscopy combined with laser induced fluorescence as diagnostic tools to observe the spectra of the Ar excited states. A schematic of the LIF system is shown in Fig. 2. As indicated in Fig. 2, a laser beam from the pulsed tunable dye laser was directed

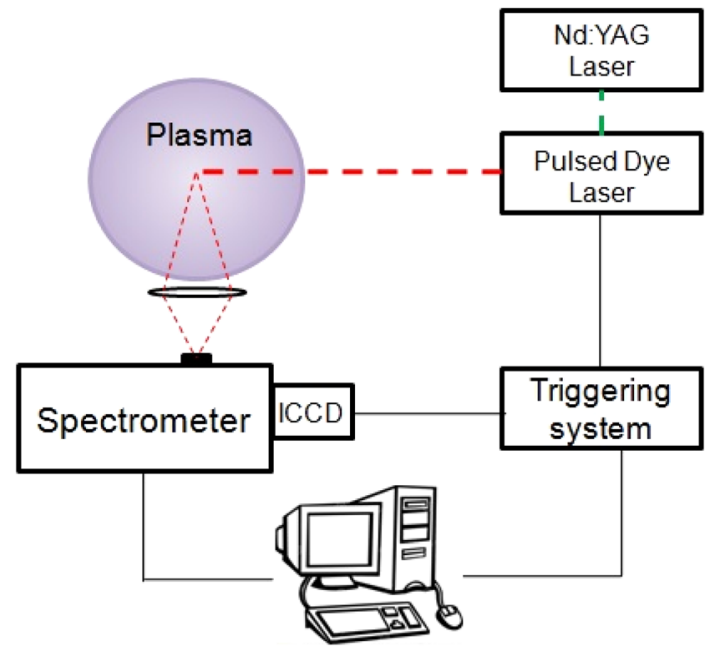

FIG. 2. Scheme of the experimental set-up using LIF diagnostics. 
into the plasmoid post discharge region to excite the atoms. The dye laser is pumped by the second harmonic of a pulsed Nd:YAG laser (Continuum, Minilite Series) operating at $532 \mathrm{~nm}$ (green light). The Nd:YAG laser light is vertically polarized with the beam diameter of $3 \mathrm{~mm}$ and $3-5 \mathrm{~ns}$ pulse width. Repetition rate of the laser was set to $f=10 \mathrm{~Hz}$ and the average power is $P=45 \mathrm{~mW}$, leading to single pulse energy of $J=4.5 \mathrm{~mJ}$. The power output of the tunable dye laser was $P_{\text {laser }}=300 \mu \mathrm{W}$ which implied the laser energy of $J_{\text {laser }}=30 \mu \mathrm{J}$. The laser beam of the pulsed dye laser was $5 \mathrm{~mm}$ in diameter. The bandwidth of the dye laser was estimated to be $3-5 \mathrm{GHz}$. On the other hand, for atomic transitions out of the metastable state in Ar, atomic line widths are of the order of $\mathrm{MHz}$, or as the case for the transition at $714 \mathrm{~nm}$ used in this case with Einstein $A$ coefficient of $6.25 \times 10^{5} \mathrm{~s}^{-1}$, sub MHz. The Doppler width at $T=1550 \mathrm{~K}$ is just under $2 \mathrm{GHz}$. While the laser bandwidth is not hugely greater than the Doppler width, it still easily encompasses the full profile and overlaps with the atomic line.

We collected data in the time resolved regime by employing an imaging spectrograph (Acton SP2750) in conjunction with the high precision Intensified ChargedCoupled Device (ICCD) camera (Princeton Instruments, PI MAX3) which allowed us to resolve spectra from the plasma on the microsecond time scale. All spectral measurements were performed side-on with respect to the direction of the discharge flow.

The supersonic discharge in this experiment was sustained by using the commercial MW generator powered on the US power electrical grid. The power signal was a $120 \mathrm{~V}$ RMS (root mean square) sine wave operating at $\nu=60 \mathrm{~Hz}$ frequency, shown in Fig. 3(a). In order to obtain timeresolved Ar I spectra, it was necessary to synchronize the detecting system (ICCD camera) with the sine power signal using a Transistor-Transistor Logic (TTL) pulse wave, as shown in Fig. 3(b). We have also synchronized the pulse of a tunable dye laser with this device to perform time-resolved laser induced fluorescence. This was achieved in the following steps. First, we reduced the supply voltage amplitude from $120 \mathrm{~V}$ to $5 \mathrm{~V}$. Second, we employed a signal inverter (74LS04) in parallel with a microcontroller (Arduino) to invert and rectify the sine signal. Further, the Arduino microcontroller emitted a TTL $15 \mu$ s pulse to trigger the Nd:YAG laser at a proper time. At the same time, another TTL signal, sent from a laser, triggered the ICCD camera. The ICCD camera was then used to collect data during a $20 \mu$ sinterval. In order to reduce the signal-to-noise ratio, we recorded 200 spectra per each exposure time.

Gas temperature of the discharge was determined in the previous work ${ }^{18}$ at the same experimental set-up. The spectrometer was positioned so that it measured spectra along the axis of symmetry for the identical discharge as in this paper. Thus, the radial location of LIF measurement coincided with the radial location of temperature measurement in Ref. 18. It can be seen that there are two time scales in this experiment. They differ by five orders of magnitude. Time duration of the microwave pulse was around $8 \mathrm{~ms}$ (as shown in Fig. 3(b)). Time duration of one point measurement that includes laser pulse duration plus delay, argon line decay, and ICCD
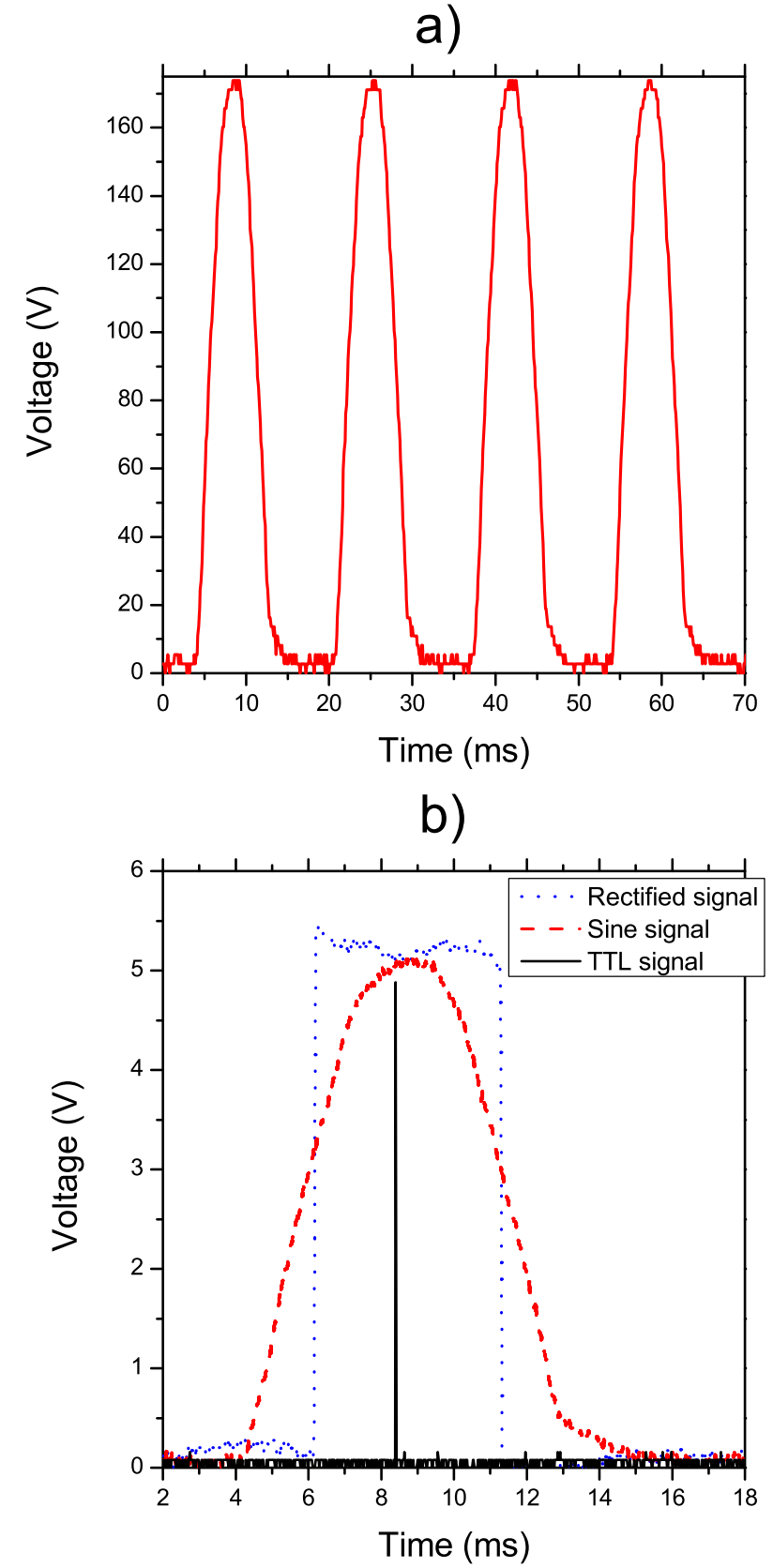

FIG. 3. Power signal: (a) at $120 \mathrm{~V}$ RMS (169.7 V peak value) and at frequency of $60 \mathrm{~Hz}$ that is equivalent to period $T=\frac{1}{\nu}=16 \mathrm{~ms}$ and (b) time synchronization between the input power signal and the detecting system.

recording time was about $20 \mu \mathrm{s}$. Within the measurement time interval, the atoms were frozen in a sphere with diameter equal to $0.1 \mu \mathrm{m}$, approximately. Therefore, Argon atoms at the velocity of $600 \mathrm{~m} / \mathrm{s}$ and at the temperature of $1550 \mathrm{~K}$ could diffuse less than $1 \mathrm{~mm}$ from the point of excitation. Thus, all emitting line spectra were within the solid angle of the ICCD detector fiber optics and were accounted for. In the space and the time scale of measurement, the temperature variations could be less than one percent, which is much less than the error in spectroscopic temperature determination from Ref. 18.

The observed spectra were calibrated using a Newport/ Oriel absolute black body irradiance source. The calibration graphs were taken under the same conditions and by 
repeating the same geometry of the experiment as the experimental data. We evaluated the calibration graphs of spectral irradiance per count versus wavelength for wavelengths between 500 and $1100 \mathrm{~nm}$. Using these graphs, we determined the population densities of the upper energy levels of particular Ar excited state transitions. A detailed procedure of how this was done is given in Ref. 17.

By using a standard back reflector procedure, we eliminated the possibility that the optical thickness of measured spectral line intensity was interfering with our measurement. All spectral line intensities were higher by a factor of two in comparison to the direct plasma observation. ${ }^{17}$ Therefore, we declared that plasma was optically thin and the radiation trapping along the observation path could be neglected.

\section{KINETIC MODEL OF ARGON}

In our work, we will use the Paschen notation for $\mathrm{Ar}$ structure due to its simplicity. Table I presents the configuration of a neutral Ar atom (Ar I) for the first 15 energy levels together with the Paschen notation for each corresponding level. The first excited, $1 s$, levels with $J=1\left(1 s_{2}\right.$ and $\left.1 s_{4}\right)$ satisfy the selection rules $(\Delta J= \pm 1)$ and decay very fast into the ground state $(J=0)$ with radiative life times of $1.96 \mathrm{~ns}$ and $8.4 \mathrm{~ns}$, respectively. ${ }^{19}$ These levels are called resonant levels. Optical transitions from the lowest levels with $J=2$ $\left(1 s_{5}\right)$ and $J=0\left(1 s_{3}\right)$ are electric dipole forbidden, making these levels metastable levels.

Emissions from Ar plasma are mostly dominated by the transitions between the first two excited configurations $(4 p$ and $4 s),{ }^{19}$ which are shown in Fig. 4. Optical emission transitions observed in our argon discharge are marked in red. For the characterization of the discharge, population densities of both upper $(4 p)$ and lower $(4 s)$ energy levels should be determined. Population densities of the upper energy levels are obtained from the intensity of the light emitted at the corresponding wavelength (spectral line emission intensity). ${ }^{17}$ However, in order to obtain population densities of the Ar I lower energy levels $(4 s)$, we have developed a simplified

TABLE I. Configuration of energy levels of Ar I with comparison to Paschen notation.

\begin{tabular}{lccc}
\hline \hline Paschen label & Level & $\mathrm{J}$ & Energy (eV) \\
\hline ground & $3 p^{6}{ }^{1} S_{0}$ & 0 & 0.0 \\
$1 s_{5}$ & $4 s[3 / 2]_{2}{ }^{3} P_{2}$ & 2 & 11.55 \\
$1 s_{4}$ & $4 s[3 / 2]_{1}{ }^{3} P_{1}$ & 1 & 11.62 \\
$1 s_{3}$ & $4 s^{\prime}[1 / 2]_{0}{ }^{3} P_{0}$ & 0 & 11.72 \\
$1 s_{2}$ & $4 s^{\prime}[1 / 2]_{1}{ }^{1} P_{1}$ & 1 & 11.83 \\
$2 p_{10}$ & $4 p[1 / 2]_{1}{ }^{3} S_{1}$ & 1 & 12.91 \\
$2 p_{9}$ & $4 p[5 / 2]_{3}{ }^{3} D_{3}$ & 3 & 13.08 \\
$2 p_{8}$ & $4 p[5 / 2]_{2}{ }^{3} D_{2}$ & 2 & 13.09 \\
$2 p_{7}$ & $4 p[3 / 2]_{1}{ }^{3} D_{1}$ & 1 & 13.15 \\
$2 p_{6}$ & $4 p[3 / 2]_{2}{ }^{3} P_{2}$ & 2 & 13.17 \\
$2 p_{5}$ & $4 p[1 / 2]_{0}{ }^{3} P_{0}$ & 0 & 13.27 \\
$2 p_{4}$ & $4 p^{\prime}[3 / 2]_{1}{ }^{1} P_{1}$ & 1 & 13.28 \\
$2 p_{3}$ & $4 p^{\prime}[3 / 2]_{2}{ }^{1} D_{2}$ & 2 & 13.30 \\
$2 p_{2}$ & $4 p^{\prime}[1 / 2]_{1}{ }^{3} P_{1}$ & 1 & 13.33 \\
$2 p_{1}$ & $4 p^{\prime}[1 / 2]_{0}{ }^{1} S_{0}$ & 0 & 13.48 \\
\hline \hline
\end{tabular}

kinetic model of argon that we combined with the OES and LIF methods.

Since our goal is to obtain population densities of $\operatorname{Ar} s$ (metastable $1 s_{5}, J=2$ and resonant $1 s_{4}, J=1$ ) states, we will concentrate on population density rates of $\operatorname{Ar} p$ states that can be determined directly from the OES method. Our assumption is a simplified model which does not include ionization and recombination processes due to their small coefficient rates and low ionization coefficient of the plasma $\left(\leq 10^{-4}\right)$. The effects of radiation trapping can be neglected since plasma is optically thin and emission lines are minimally affected by reabsorption phenomena. Furthermore, our model did not include any spatial variations in the population densities. We applied the model to the emissions from the center of the plasma volume. In general, the model could be applied to describe emissions from any volume element in plasma by taking into account the effects that are spatial dependent. ${ }^{19}$ According to the above assumptions, population rate of $\operatorname{Ar} 2 p_{x}(x=1,2, \ldots)$ excited levels are mainly affected by the following processes: ${ }^{8,19-21}$

(1) Electron excitation from the ground state

$$
e+\operatorname{Ar}\left(N_{n}\right) \rightarrow e+\operatorname{Ar}\left(N_{p x}\right) .
$$

(2) Electron excitation from the metastable and resonant levels

$$
e+\operatorname{Ar}\left(N_{s m}\right) \rightarrow e+\operatorname{Ar}\left(N_{p x}\right)
$$

(3) Radiative transition from higher energy levels (4d, $5 s$, $6 s, \ldots)$

$$
\operatorname{Ar}\left(N_{\text {higher }}\right) \rightarrow \operatorname{Ar}\left(N_{p x}\right)+h \nu
$$

(4) Radiative transition to metastable and resonant levels

$$
\operatorname{Ar}\left(N_{p x}\right) \rightarrow \operatorname{Ar}\left(N_{s m}\right)+h \nu
$$

(5) Quenching (collisional deactivations)

$$
\operatorname{Ar}\left(N_{n}\right)+\operatorname{Ar}\left(N_{p x}\right) \rightarrow \operatorname{Ar}\left(N_{n}\right)+\operatorname{Ar}\left(N_{s m}\right) .
$$

Note that due to the short radiative lifetimes $(<10 \mathrm{~ns})$ of the $1 s_{2}$ and $1 s_{4}$ resonant states, their populations are much lower than the population of the $1 s_{3}$ and $1 s_{5}$ metastable states. Therefore, electron impact excitation from resonant states was neglected in this approximation. Further, the contributions from higher excited states of Ar were excluded from the model since the emissions from these states are reduced due to the low density of high-energy electrons in the plasma that are able to excite them. ${ }^{20,21}$

The net population rate of the $\operatorname{Ar}\left(N_{p x}\right)$ state is, then, given by

$$
\begin{aligned}
\frac{d N_{p x}}{d t}= & k_{e}^{g} N_{e} N_{n}+k_{e}^{s 3} N_{e} N_{s 3}+k_{e}^{s 5} N_{e} N_{s 5} \\
& -\sum_{m=2}^{5} \frac{1}{\tau_{s m}} N_{p x}-k_{c}^{g, p x} N_{n} N_{p x}
\end{aligned}
$$

where $N_{e}$ is the electron density, $N_{n}$ is the ground state population density, $N_{s i}$ are the population densities of $\operatorname{Ar}$ I $s$ 


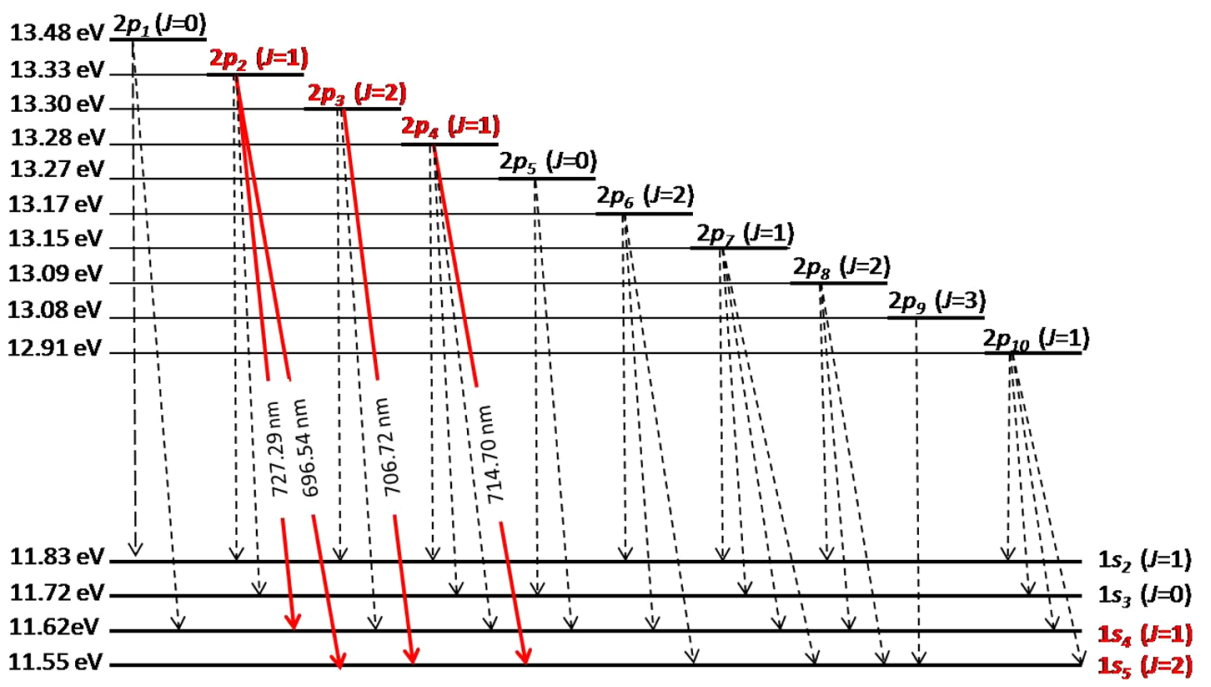

FIG. 4. Partial energy level diagram for Ar I excited states ( $4 p$ and $4 s$ ). Transitions from and to the ground state are not included in this figure. Optical emission transitions observed in our argon discharge are marked in red.

Ground state $(J=0)$

states, $k_{e}^{j}$ are rate coefficients for electric impact excitation, $k_{c}^{g, p x}$ is the rate coefficient for the collisional quenching between the atoms in ground state and excited $p$-states, and $\tau_{s m}=\frac{1}{A_{s m}}$ are the corresponding radiative lifetimes, where $A_{s m}$ are Einstein coefficients for spontaneous emission.

Most of the results in the literature included only steady state cases $\left(\frac{d N_{p x}}{d t}=0\right)$ in low pressure discharges (mTorr pressure range) when radiative decay is the main depopulation process and quenching processes could be neglected. ${ }^{8,12,20,21}$ The working pressure in our experiment was between 1 and 3 Torr suggesting that collisional quenching process involving neutrals becomes important and should be included in the model. Moreover, we derive the equation for the steady state solution but also propose a new approach for the timedependent evaluations of Ar metastable $\left(1 s_{5}\right)$ and resonant $\left(1 s_{4}\right)$ states by applying LIF.

A schematic diagram of the processes that are included in the kinetic model is shown in Fig. 5. We neglected effects of laser stimulated emission by assuming that atomic excitation is in the linear regime away from saturation, consistent with our laser parameters and experimental conditions as well as the low measured excited state fraction.

When the observed level is pumped by a laser from the desired resonant or metastable level, the new balance equation looks similar to Eq. (6) with the addition of an extra term corresponding to the laser absorption

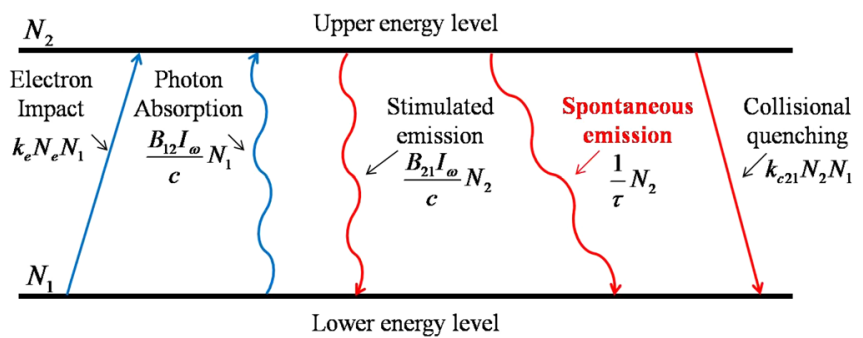

FIG. 5. Two energy level diagram describing argon kinetic model.

$$
\begin{aligned}
\frac{d N_{p x}^{\prime}}{d t}= & k_{e}^{g} N_{e} N_{n}+k_{e}^{s 3} N_{e} N_{s 3}+k_{e}^{s 5} N_{e} N_{s 5} \\
& -\sum_{m=2}^{5} \frac{1}{\tau_{s m}} N_{p x}^{\prime}-k_{c}^{g, p x} N_{n} N_{p x}^{\prime}+\frac{B_{12} I_{\omega}}{c} N_{s m},
\end{aligned}
$$

where $N_{p x}^{\prime}$ is the population density of the investigated $\operatorname{Ar} p$ state when plasma is pumped by a laser, $B_{12}$ is the Einstein coefficient for laser absorption, and $I_{\omega}$ is the laser spectral irradiance. In calculating $I_{\omega}$, we assume a laser bandwidth of $4 \mathrm{GHz}$. The uncertainty in bandwidth limits the precision of our population density calibration. For the results shown in the remainder of the paper, uncertainty of the laser bandwidth is not included in the error estimation. This uncertainty means that the values of the metastable population densities shown are only accurate to within a factor of $\sim 2$, but this uncertainty does not change the shape of the time evolution of the population.

The laser pulse duration was $3-5 \mathrm{~ns}$, and measurements were taken in $20 \mu$ s time intervals. We assumed that populations of metastable and resonant levels remained constant during each $20 \mu$ s time interval. After subtracting Eq. (6) from Eq. (7) we obtained

$$
\begin{aligned}
\frac{d}{d t}\left(N_{p x}^{\prime}-N_{p x}\right)= & -\sum_{m=2}^{5} \frac{1}{\tau_{s m}}\left(N_{p x}^{\prime}-N_{p x}\right) \\
& -k_{c}^{g, p x} N_{n}\left(N_{p x}^{\prime}-N_{p x}\right)+\frac{B_{12} I_{\omega}}{c} N_{s m} .
\end{aligned}
$$

Hence, the population of the resonant/metastable level is

$$
\begin{aligned}
\frac{B_{12} I_{\omega}}{c} N_{s m}= & \frac{d}{d t}\left(N_{p x}^{\prime}-N_{p x}\right)+\left(\sum_{m=2}^{5} \frac{1}{\tau_{s m}}+k_{c}^{g, p x} N_{n}\right) \\
& \times\left(N_{p x}^{\prime}-N_{p x}\right) .
\end{aligned}
$$




$$
N_{s m}=\frac{c \cdot\left(\sum_{m=2}^{5} \frac{1}{\tau_{s m}}+k_{c}^{g, p x} N_{n}\right)}{B_{12} I_{\omega}}\left(N_{p x}^{\prime}-N_{p x}\right)
$$

that can be solved analytically. It could be seen from Eqs. (9) and (10) that the population densities of resonant/ metastable argon levels are directly proportional to the difference between spectral line intensities (population densities of $N_{p x}$ levels) when the laser is on $\left(N_{p x}^{\prime}\right)$ and when the laser is off $\left(N_{p x}\right)$.

\section{RESULTS AND DISCUSSION}

Time resolved measurements were taken $5 \mathrm{~cm}$ downstream of the cavity in the experiment, which corresponds to the middle area of the plasmoid, shown in Fig. 1. The working pressure was set to $p=2.3$ Torr.

\section{A. Population densities of Ar I $2 p$ levels}

We have obtained population distributions of three Ar I excited $p$ states:

- $2 p_{2}$ or $4 p^{\prime}[1 / 2]_{1}{ }^{3} P_{1} \quad J=1$ state from the spectral line intensity at $696.54 \mathrm{~nm}$

- $2 p_{3}$ or $4 p^{\prime}[3 / 2]_{2}{ }^{1} P_{2} J=2$ state from the spectral line intensity at $706.72 \mathrm{~nm}$

- $2 p_{4}$ or $4 p^{\prime}[3 / 2]_{1}{ }^{1} P_{1} \quad J=1$ state from the spectral line intensity at $714.70 \mathrm{~nm}$.

Figure 6 shows the time dependent population distributions compared to the power sine signal. The estimated measurement errors originate from the statistical error in data acquisition and were no more than $7 \%$. It can be deduced from the figure that during and immediately after the breakdown (the first two milliseconds), population distributions peak in their values. This is followed by the decrease in intensities during the plasma stabilization. Finally, when the discharge becomes quasi-stationary, the population density distributions show weak time dependence and can be approximated with their average values. The variation of the average values is probably due to additional changes of plasma conditions that are not included in the error estimation. Peak population densities are determined to be $N_{p 2}^{\text {peak }}=(4.8 \pm 0.5) \times 10^{5} \mathrm{~cm}^{-3}$, $N_{p 3}^{\text {peak }}=(5.0 \pm 0.4) \times 10^{5} \mathrm{~cm}^{-3}, \quad$ and $\quad N_{p 4}^{\text {peak }}=(7.8 \pm 0.6)$ $\times 10^{5} \mathrm{~cm}^{-3}$, while average values of population densities are estimated to $N_{p 2}^{a v}=1.0 \times 10^{5} \mathrm{~cm}^{-3}, N_{p 3}^{a v}=1.1 \times 10^{5} \mathrm{~cm}^{-3}$, and $N_{p 4}^{a v}=1.6 \times 10^{5} \mathrm{~cm}^{-3}$ for Ar I excited states $2 p_{2}, 2 p_{3}$, and $2 p_{4}$, respectively.

The average values of population densities obtained using this method are in good agreement with the values of the population densities obtained using the 2D Plasma Tomography method. ${ }^{16}$ This implies that both techniques (time resolved OES and Plasma Tomography) provide not only a qualitative but also a quantitative description of the plasmoid object.
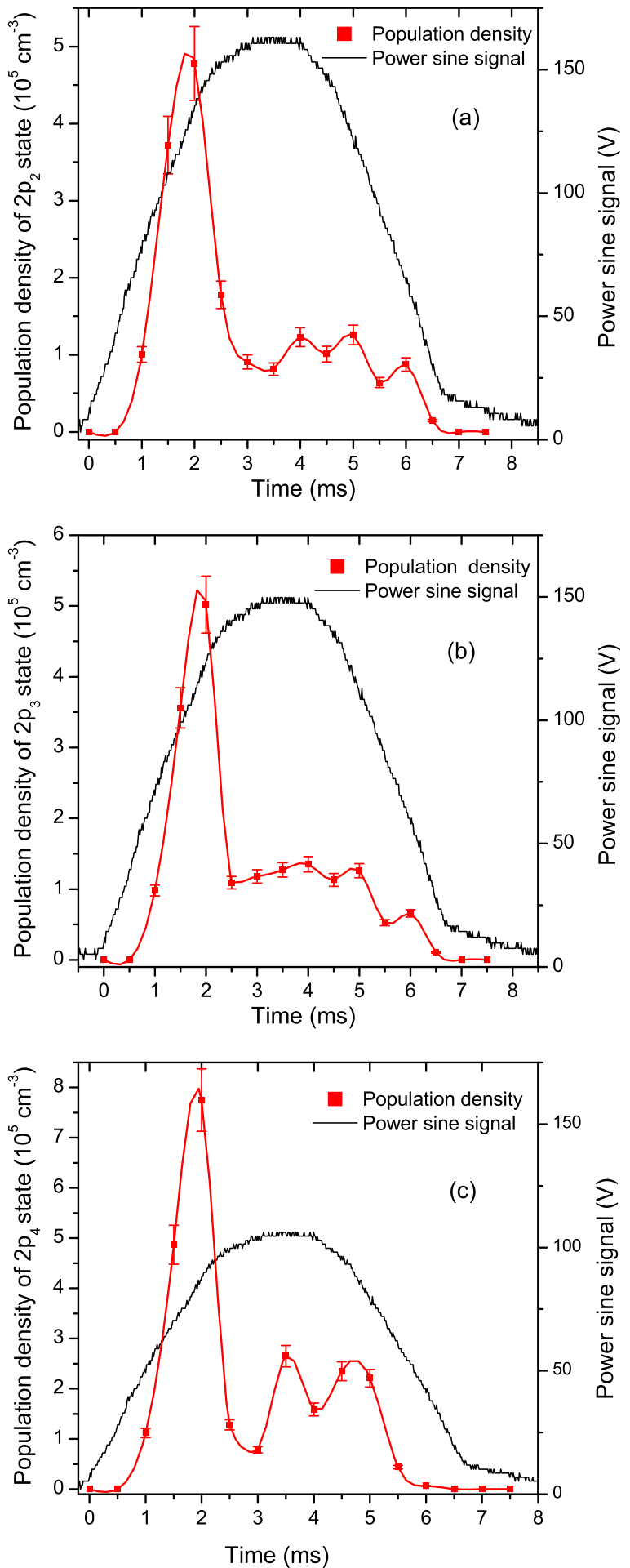

FIG. 6. Time dependence of the Ar I population densities at 2.3 Torr: (a) $2 p_{2}$ state, (b) $2 p_{3}$ state, and (c) $2 p_{4}$ state. Error bars are indicated. As a guideline, data are connected with cubic spline fit. Microwave waveform is presented to illustrate variation of the population density.

\section{B. Population densities of Ar I $1 s$ levels}

We have obtained population distributions of two Ar I excited $s$ states:

- Metastable $1 s_{5}$ or $4 s[3 / 2]_{2}{ }^{3} P_{2} J=2$ state from the spectral line intensity at $706.72 \mathrm{~nm}$

- Resonant $1 s_{4}$ or $4 s[3 / 2]_{1}{ }^{3} P_{1} J=1$ state from the spectral line intensity at $727.29 \mathrm{~nm}$. 
Argon metastable/resonant level population densities were obtained by applying an Ar kinetic model, in the following way. First, we calculated the difference in intensities of measured Ar I $2 p$ states with and without presence of a dye laser light beam. Further, we determined the first derivative of the calculated difference. These results were used to evaluate time-resolved population densities of metastable/ resonant states by employing Eq. (9).

Time-resolved population densities of Ar I metastable $1 s_{5}$ level were determined from the kinetic equation of the Ar I $2 p_{3}$ level, derived from Eq. (9)

$$
\begin{aligned}
\frac{B_{12} I_{\omega}}{c} N_{s 5}= & \frac{d}{d t}\left(N_{p 3}^{\prime}-N_{p 3}\right)+\left(\frac{1}{\tau_{s 2}}+\frac{1}{\tau_{s 4}}+\frac{1}{\tau_{s 5}}+k_{c}^{g, p 3} N_{n}\right) \\
& \times\left(N_{p 3}^{\prime}-N_{p 3}\right),
\end{aligned}
$$

where $\tau_{s 2}=7.8 \mathrm{~ns}, \tau_{s 4}=23.5 \mathrm{~ns}$, and $\tau_{s 5}=52.6 \mathrm{~ns}$ are radiative life times of the Ar I $2 p_{3}$ level to $1 s_{2}, 1 s_{4}$, and $1 s_{5}$ levels, respectively.

We have obtained time-resolved population densities of Ar I metastable $1 s_{5}$ level at two different pressures, 1.7 Torr and 2.3 Torr. Ground state population densities, $N_{n}$, necessary for evaluation of Ar I metastable $1 s_{5}$ populations were obtained from the ideal gas equation

$$
N_{n}=\frac{p}{k T_{g}},
$$

where $T_{g}$ is gas temperature determined in the work of Drake et al. ${ }^{18}$ They have observed that in the range of pressures between 1.5 and 2.5 Torr, gas temperature varies weakly with pressure around $T_{g}=1500 \pm 200 \mathrm{~K}$. Temperature drop along the axis was measured to be $100 \mathrm{~K} / \mathrm{m}$, which was taken into account in the error estimation in gas temperature used for the kinetic model. This error was included in the measured result (shown by the error bars in Figs. 7 and 8). Thus, ground state population densities were estimated to $N_{n}=1.1$ $\pm 0.210^{16} \mathrm{~cm}^{-3}$ at 1.7 Torr and $N_{n}=1.4 \pm 0.310^{16} \mathrm{~cm}^{-3}$ at 2.3 Torr.

Rate coefficient for the collisional quenching $k_{c}^{g, p 3}$ was calculated by employing the equation ${ }^{22}$

$$
\begin{aligned}
k_{c}^{g, p 3}\left(\mathrm{~cm}^{-3} / \mathrm{s}\right) & =3 \times 10^{-11}\left(\frac{T_{g}}{300}\right)^{0.5} \\
& =6.8 \times 10^{-11} \mathrm{~cm}^{-3},
\end{aligned}
$$

where $T_{g}=1500 \pm 200 \mathrm{~K}$.

Einstein coefficient for laser absorption was determined from the well known equations for blackbody radiation ${ }^{23}$

$$
B_{12}=\frac{g_{2}}{g_{1}} \frac{A_{21} \lambda^{3}}{8 \pi h} .
$$

For the statistical weight of the $1 s_{5}$ metastable level of $g_{s 5}$ $=5$ and the transition wavelength $\lambda=706 \mathrm{~nm}$, the Einstein absorption coefficient is equal to $B_{12}=8.5 \times 10^{19} \mathrm{~J}^{-1} \mathrm{~m}^{3} \mathrm{~s}^{-2}$.

Time variations of metastable population densities obtained at two pressures are shown in Fig. 7. Experimental errors presented in the figure were estimated from the

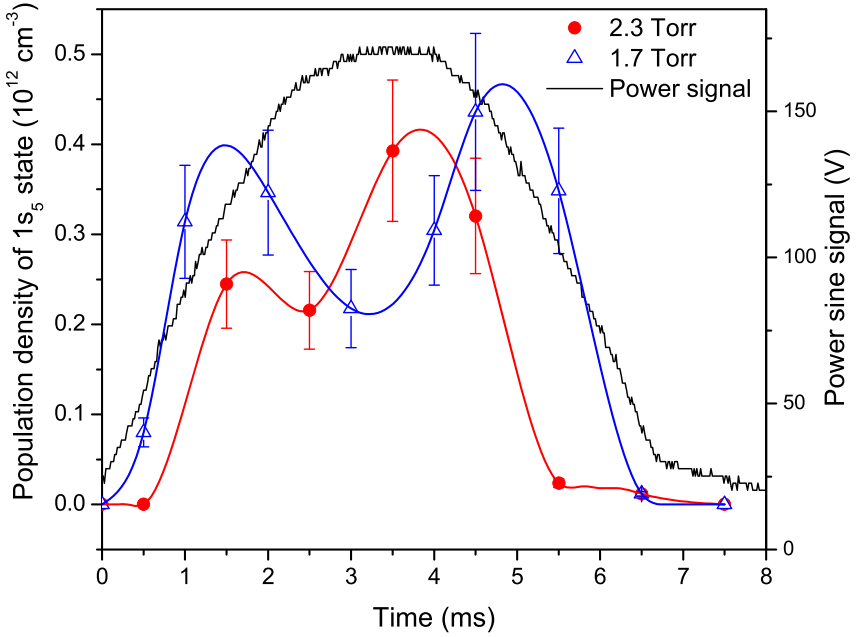

FIG. 7. Time dependence of the Ar I population densities of the metastable $1 s_{5}$ state. Data are taken at indicated pressures. Error bars are indicated and reflect the statistical variations in measured values. As a guideline, data are connected with cubic spline fit. Microwave waveform is presented to illustrate variation of the population density.

experimental errors in obtaining population densities of $\mathrm{Ar}$ I $2 p_{3}$ level and corresponding first derivative calculations. They were determined to be within $20 \%$.

It could be seen from the figure that at both pressures metastable states have two peaks within the power signal. It seems as if the population densities oscillate at twice the frequency of the power signal $(f=120 \mathrm{~Hz})$. A similar effect has already been observed by Macdonald et al $^{24}$ in the case of a xenon discharge lamp. The reasons for this effect, which is reminiscent of the frequency doubling, may lay in the geometry of the surface wave, local E-field variation, and the kinetics of the discharge. Future work will elucidate this effect.

However, Fig. 7 indicates that at lower pressure metastable population densities peak in their values $1.5 \mathrm{~ms}$ after the beginning of the signal and just before the end of the sine signal $(5 \mathrm{~ms}$ after the beginning of the signal), while at

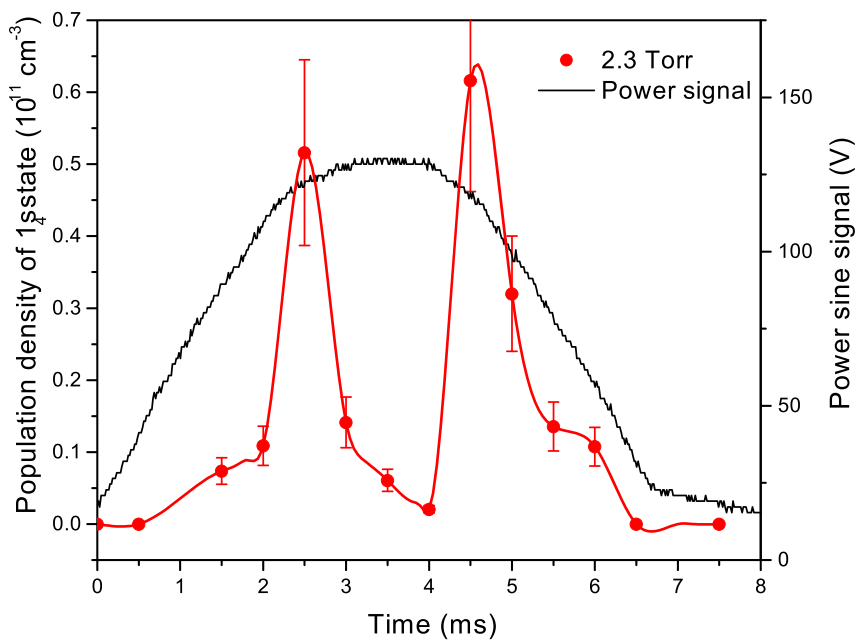

FIG. 8. Time dependence of the Ar I population densities of the Ar I resonant $1 s_{4}$ state. Data are taken at indicated pressure. Error bars are indicated. As a guideline, data are connected with cubic spline fit. Microwave waveform is presented to illustrate variation of the population density. 
higher pressure the first peak occurs at a later time $(1.7 \mathrm{~ms}$ after the beginning of the signal) and the second peak occurs $4 \mathrm{~ms}$ after the beginning of the signal. This could be explained by the fact that at higher pressures collisional quenching processes contribute more to the kinetics of $\mathrm{Ar}$ discharge, which leads to the lower population densities.

Similarly, we have obtained time-resolved population densities of Ar I resonant $1 s_{4}$ level by employing the kinetic equation of the $\operatorname{Ar} I 2 p_{2}$ level

$$
\begin{aligned}
\frac{B_{12} I_{\omega}}{c} N_{s 4}= & \frac{d}{d t}\left(N_{p 2}^{\prime}-N_{p 2}\right) \\
& +\left(\frac{1}{\tau_{s 2}}+\frac{1}{\tau_{s 3}}+\frac{1}{\tau_{s 4}}+\frac{1}{\tau_{s 5}}+k_{c}^{g, p 2} N_{n}\right)\left(N_{p 2}^{\prime}-N_{p 2}\right),
\end{aligned}
$$

where $\tau_{s 2}=24.0 \mathrm{~ns}, \tau_{s 3}=19.9 \mathrm{~ns}, \tau_{s 4}=1.5 \times 10^{4} \mathrm{~ns}$, and $\tau_{s 5}=532 \mathrm{~ns}$ are radiative life times of the Ar I $2 p_{2}$ level to $1 s_{2}, 1 s_{3}, 1 s_{4}$, and $1 s_{5}$ levels, respectively.

In Fig. 8 we present time-resolved population densities of Ar I resonant $1 s_{4}$ level at pressure of 2.3 Torr. Rate coefficient for the collisional quenching $k_{c}^{g, p 2}$ was calculated by employing the equation ${ }^{22}$

$$
\begin{aligned}
k_{c}^{g, p 2}\left(\mathrm{~cm}^{-3} / \mathrm{s}\right) & =1 \times 10^{-11}\left(\frac{T_{g}}{300}\right)^{0.5} \\
& =2.3 \times 10^{-11} \mathrm{~cm}^{-3}
\end{aligned}
$$

where $T_{g}=1500 \pm 200 \mathrm{~K}$.

The Einstein coefficient for laser absorption was equal to $B_{12}=4.5 \times 10^{19} \mathrm{~J}^{-1} \mathrm{~m}^{3} \mathrm{~s}^{-2}$ where the statistical weight of the $1 s_{4}$ metastable level was $g_{s 4}=3$ and the transition wavelength was $\lambda=727 \mathrm{~nm}$.

Figure 8 shows time dependence of Ar I resonant $1 s_{4}$ population densities at pressure of 2.3 Torr. We have observed similar behavior as for the metastable states. Resonant states also have two peaks within the power signal oscillating at twice the frequency of the power signal. Experimental errors were estimated the same way as in the case of metastable population densities. They were determined to be within $20 \%$, again neglecting laser bandwidth uncertainty.

It should be noted that the first derivatives of the Ar I $2 p_{2}$ and $2 p_{3}$ levels showed similar time dependence with and without the laser signal. This implies that their difference can be approximated to zero. Thus, the simplified case given by Eq. (10) can be applied within the $20 \%$ error.

\section{v. CONCLUSION}

A technique for evaluation of time-resolved population densities of Ar I metastable $1 s_{5}$ and resonant $1 s_{4}$ levels was developed and demonstrated. The technique employs the kinetic model of Ar based on the analysis of population density rates of $\operatorname{Ar} I p$ states combined with the Laser Induced
Fluorescence method. The method was tested in supersonic flowing microwave discharge in argon. LIF measurements were performed by using the tunable dye laser that operates in the infrared region and is pumped by the second harmonic of a pulsed NdYag laser operating at $532 \mathrm{~nm}$. The pump laser was synchronized with the microwave power supply and the detection system using the synchronization device providing the time-resolved measurements on a microsecond time scale. We obtained population distributions of two $\mathrm{Ar}$ I excited $s$ states: metastable $1 s_{5}$ or $4 s[3 / 2]_{2}{ }^{3} P_{2} J=2$ state from the spectral line intensity at $706.72 \mathrm{~nm}$ and resonant $1 s_{4}$ or $4 s[3 / 2]_{1}^{3} P_{1} J=1$ state from the spectral line intensity at $727.29 \mathrm{~nm}$. It seems that population densities of Ar I metastable $1 s_{5}$ and resonant $1 s_{4}$ levels oscillate with twice the frequency of the power signal, which was indicated by two peaks within the power signal.

\section{ACKNOWLEDGMENTS}

This work was supported by the Office of the High Energy Physics, Office of Science, Department of Energy under Grant No. DE-SC0007879.

${ }^{1}$ D. B. Graves, AIChE 35, 1 (1989).

${ }^{2}$ M. J. W. Coburn, Plasma Chem. Plasma Process. 2, 1 (1982).

${ }^{3}$ D. M. Manos and D. L. Flamm, Plasma Etching: An Introduction (Academic Press, New York, 1989).

${ }^{4}$ M. Konuma, Film Deposition by Plasma Techniques (Springer, New York, 1992).

${ }^{5}$ M. A. Lieberman and A. J. Lichtenberg, Principles of Plasma Discharges and Materials Processing (Academic Press, New York, 1994).

${ }^{6}$ M. A. Lieberman and R. A. Gottscho, Design of High Density Plasma Sources for Materials Processing (Wiley, New York, 1994).

${ }^{7}$ V. Guerra, P. A. Sa, and J. Loureiro, Eur. Phys. J. Appl. Phys. 28, 125 (2004).

${ }^{8}$ V. M. Donnelly, J. Phys. D: Appl. Phys. 37, R217 (2004).

${ }^{9}$ G. A. Hebner, J. Appl. Phys. 80, 2624 (1996).

${ }^{10}$ B. K. Mcmillin and M. R. Zachariah, J. Appl. Phys. 79, 77 (1996).

${ }^{11}$ N. Beverini, G. Cicconi, G. L. Genovesi, and E. Piano, Plasma Sources Sci. Technol. 6, 185 (1997).

${ }^{12}$ A. Bogaerts and R. Gijbels, Phys. Rev. A 52, 3743 (1995).

${ }^{13}$ M. W. Kiehlbauch and D. B. Graves, J. Appl. Phys. 91, 3539 (2002).

${ }^{14}$ S. Rauf and M. J. Kushner, J. Appl. Phys. 82, 2805 (1997).

${ }^{15}$ E. A. D. Carbone, S. Hübner, J. J. A. M. van der Mullen, G. M. W. Kroesen, and N. Sadeghi, J. Phys. D: Appl. Phys. 46, 415202 (2013).

${ }^{16}$ M. Nikolić, A. Samolov, F. Čučkov, S. Popović, L. Vušković, and A. Godunov, J. Appl. Phys. 113, 103301 (2013).

${ }^{17}$ M. Nikolić, Ph.D. dissertation, Old Dominion University, 2013.

${ }^{18}$ D. J. Drake, S. Popović, and L. Vušković, J. Appl. Phys. 104, 063305 (2008).

${ }^{19}$ J. Boffard, R. O. Jung, C. C. Lin, and A. E. Wend, Plasma Sources Sci. Technol. 19, 065001 (2010).

${ }^{20}$ J. Boffard, C. C. Lin, and C. A. De Joseph, Jr., J. Phys. D: Appl. Phys. 37, R143 (2004).

${ }^{21}$ D. Mariotti, Y. Shimizu, T. Sasaki, and N. Koshizaki, Appl. Phys. Lett. 89, 201502 (2006).

${ }^{22}$ X. M. Zhu and Y. K. Pu, J. Phys. D: Appl. Phys. 43, 015204 (2010).

${ }^{23}$ R. Laudon, The Quantum Theory of Light (University Press, Oxford, 2000).

${ }^{24}$ N. A. MacDonald, M. A. Capelli, and W. A. Hargus, Jr., Rev. Sci. Instrum. 83, 113506 (2012). 